\title{
Time-aware Location Sequence Recommendation for Cold-start Mobile Users
}

\author{
Ting Shen \\ Auburn University \\ Auburn, AL, USA \\ ting@auburn.edu
}

\author{
Haiquan Chen \\ California State University \\ Sacramento, CA, USA \\ haiquan.chen@csus.edu
}

\author{
Wei-Shinn $\mathrm{Ku}$ \\ Auburn University \\ Auburn, AL, USA \\ weishinn@auburn.edu
}

\begin{abstract}
In this paper, we study the problem of recommending time-sensitive location sequence for mobile users using their check-in data on location-based social networks. Most of the existing studies on Point of Interest (POI) recommendation and prediction fail to address the following two key challenges: (1) how to handle the scenario where the user-location matrix is very sparse (i.e., each user has a very limited number of check-ins, or to say, cold-start users), and (2) how to recommend an optimal time-sensitive visit sequence where each venue matches a time slot specified by users, based on their check-in histories. Motivated by the two challenges above, we propose a predictive framework that enables time-sensitive location sequence recommendation leveraging both the users' semantic and spatial similarities, especially for cold-start users. Our novel framework consists of three modules: semantic similarity modeling, spatial similarity modeling, and on-line sequence recommendation. In semantic modeling, we calculate users' similarity scores by comparing users' temporal hierarchical semantic trees. In spatial modeling, we use Gaussian Mixture Model (GMM) to compute users' similarity scores with respect to their geographical movement patterns. Afterwards, we combine the check-in data of the target user with those of her top- $k$ most similar users in terms of both semantic and spatial similarities to train a personalized Hidden Markov Model (HMM) to predict the most probable venue category for each specified time slot. At last, we recommend location sequence based on the predicted venue category sequence for the target user using geographical mapping.
\end{abstract}

\section{CCS CONCEPTS}

-Information systems $\rightarrow$ Location based services;

\section{KEYWORDS}

Location-based social networks, hidden Markov models

\section{ACM Reference format:}

Ting Shen, Haiquan Chen, and Wei-Shinn Ku. 2018. Time-aware Location Sequence Recommendation for Cold-start Mobile Users. In Proceedings of 26th ACM SIGSPATIAL International Conference on Advances in Geographic Information Systems, Seattle, WA, USA, November 6-9, 2018 (SIGSPATIAL '18), 4 pages.

DOI: $10.1145 / 3274895.3274958$

Permission to make digital or hard copies of part or all of this work for personal or classroom use is granted without fee provided that copies are not made or distributed for profit or commercial advantage and that copies bear this notice and the full citation on the first page. Copyrights for third-party components of this work must be honored. For all other uses, contact the owner/author(s).

SIGSPATIAL '18, Seattle, WA, USA

(C) 2018 Copyright held by the owner/author(s). $978-1-4503-5889-7 / 18 / 11 \ldots \$ 15.00$ DOI: $10.1145 / 3274895.3274958$

\section{INTRODUCTION}

With the rapid development of positioning technologies and personal devices such as smart-phones, a number of Location-based Social Networks (LBSNs) have emerged, e.g., Foursquare and GeoLife. With these applications, users are able to share their location data. These records imply extensive knowledge about a user's interests and behavior, and mining such data has become an interesting research topic. Check-in records have two very important components, the location and the time. Because of the particularity of check-in record, it is possible to analyze user's action according to the user's mobility and activities in the physical world.

A location recommendation often simultaneously considers the following factors. (1) User record. (2) Behavior of other users. (3) User's current location. Most of the existing location-based recommender systems have the following limitations: (1) providing a list of separate locations which match a user's personal interest without the support of continuous location sequence recommendation[12] , (2) requiring a large amount of check-in data from the target user and other users to make the predictive systems work (i.e., cold-start problem), (3) inability to consider temporal factors when making venue recommendation because users' preferences on venues are actually time-dependent or time-sensitive. In this paper, we aim to develop a novel framework for recommending an optimal timesensitive visit sequence that best matches the time slots specified by a target user.

By taking full advantage of the similarities between mobile users, our proposed framework does not suffer from the cold-start problem and works well even if the target user has very few footprints (check-ins) on a LBSN site. The key contributions of this paper are as follows:

- We propose a predictive framework that enables timesensitive location sequence recommendation leveraging both users' semantic and spatial similarities in support of sparse user-location matrix. Our novel framework consists of three modules: semantic similarity modeling, spatial similarity modeling, and on-line sequence recommendation.

- In semantic similarity modeling, we build a venue categorical semantic tree, i.e., Weighted Category Hierarchy (WCH), to capture each user's venue categorical preference based on users' check-in histories. Given any two users, their semantic similarity score can be calculated as the weighted sum of the Jensen-Shannon divergence of each level in their corresponding Normalized Weighted Category Hierarchies (NWCHs). 
- In spatial similarity modeling, we employ Gaussian Mixture Model (GMM) to capture users' geographical movement patterns. The in-between user spatial similarity scores can then be estimated by computing the average log-likelihood that a user's check-in records fit with the GMMs learned from other users.

- Given any target user, we train her personalized Hidden Markov Model (HMM) by combining the check-in records from herself and her top- $k$ most semantically and spatially similar users, identified in the above semantic and spatial similarity modeling. Such trained personalized HMM will then be used to predict the most probable venue categories for all the specific time slots and recommend a location sequence to the target user based on geographical mapping.

\section{OVERVIEW OF OUR APPROACH}

In this section, we introduce the motivating example and the architecture of our time-aware location sequence recommender system.

In a location-based social network, a user can visit multiple locations, which may generate a check-in sequence. The user's check-in records reflect her location history in the real world. As illustrated in Figure 1, the system maintains a sequence of checkin records for each user. Each check-in record shows the time when the user checked in and the venue where the user visited. A venue check-in contains the information of the real-world location (latitude and longitude) that can be shown on a map, associated with a set of categories. In Foursquare the categories are represented by a category tree. For example, category "Food" includes category "Chinese Restaurant" and "American Restaurant", and "Chinese Restaurant" includes "Cantonese Restaurant".

Our system allows a user to set the time schedule by entering a sequence of time slots when she wants to visit a few venues. The length of the sequence is decided by the user. For example, a user may choose 10:00, 12:00, and 13:00 as the three time slots. Then she specifies the second venue she wants to visit must be a restaurant. The system will generate a sequence of venues that best matches her selections based on both the check-in histories of herself and her top- $k$ most similar users. Figure 2 shows the system architecture, which consists of offline modeling, online recommendation and user interface.

\section{OFFLINE TRAINING}

In this section, we introduce the offline modeling of our system, which is composed of two modules: 1) the semantic similar user

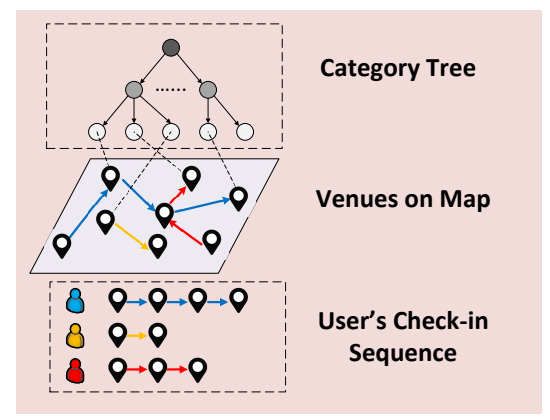

Figure 1: User Check-ins on Social Network. discovery, which generates a semantic tree for each user and computes their semantic similarity scores, 2) the spatial similar user discovery, which fits a Gaussian mixture model with each user's check-in records and then calculates users' spatial similarity scores, and 3) personalized HMM training, which trains a hidden Markov model for each user.

\subsection{User Semantic Similarity}

This module first extracts a user's semantic preference based on the categories of her visited places. Figure 3 shows the process of calculating a user's weighted category hierarchy (WCH) [3]. In a $\mathrm{WCH}$, each node is associated with the frequency of a user's checkins at the corresponding category. In such hierarchy, a deeper level denotes the categories of a finer granularity. Next, an Inverse Document Frequency (IDF) tree will be calculated. As shown in the Figure 3, the value of each node represents the IDF value of the category. At last, the two values for the same node will be multiplied. Equation 1 shows how to compute the TF-IDF value of category c' in the hierarchy of user u(i.e., $\left.u . w_{c^{\prime}}\right)$.

$$
u \cdot w_{c^{\prime}}=\frac{\left|\left\{u \cdot v_{i}: v_{i} \cdot c=c^{\prime}\right\}\right|}{u \cdot \mathcal{V}} \times \lg \frac{|\mathcal{U}|}{\left|\left\{u_{j} \cdot c^{\prime} \in u_{j} \cdot C\right\}\right|}
$$

The first term of the equation is the TF value, where $\mid\left\{u . v_{i}:\right.$ $\left.v_{i} . c=c^{\prime}\right\} \mid$ is user $u$ 's number of visits to venues in category c', and $u . \mathcal{V}$ is the user $u$ 's total number of visits. The second term of the equation is IDF value, where $|\mathcal{U}|$ is the number of all the users and $\left|\left\{u_{j} . c^{\prime} \in u_{j} . C\right\}\right|$ is the number of users who have visited category c'.

Such a $\mathrm{WCH}$ is able to capture an individual's preferences in different categories of different granularity. For example, the user shown in Figure 3 has interest in "Food" and "Entertainment", and in the category "Food" she likes "Chinese Restaurant".

To compute the similarity score of any two users based on their WCHs, the naive method is simply counting the overlapped nodes (check-in occurrences) at each venue category. For cold-start users, this approach leads to many close to zero or zero weights, leading to a very poor performance of the similarity comparison in practice. To solve the problem, we employ the Jensen-Shannon divergence[7], which is originally defined as follows:

$$
\mathrm{JSD}(P \| Q)=\frac{1}{2} \sum P\left(\log \frac{P}{M}\right)+\frac{1}{2} \sum Q\left(\log \frac{Q}{M}\right)
$$

with $M=\frac{1}{2}(P+Q)$ where $P$ and $Q$ are two probabilist distributions. We normalized each WCH into Normalized WCH (NWCH) by normalizing all the IT-IDF scores for each category at the same level so that they sum to 1 . The semantic similarity score of two users can then be calculated as the weighed sum of the J-S divergence of each level in the two corresponding $\mathrm{NWCH}$, i.e., the semantic trees. For each user $u_{i}$, we keep a list of the users with the top- $k$ highest semantic similarity scores to her.

\subsection{User Spatial Similarity}

Besides the preferences on venue categories, the users also have preferences on different geographical areas. To extract a user's spatial preference, we use Gaussian Mixture Model (GMM) to cluster check-in locations. 


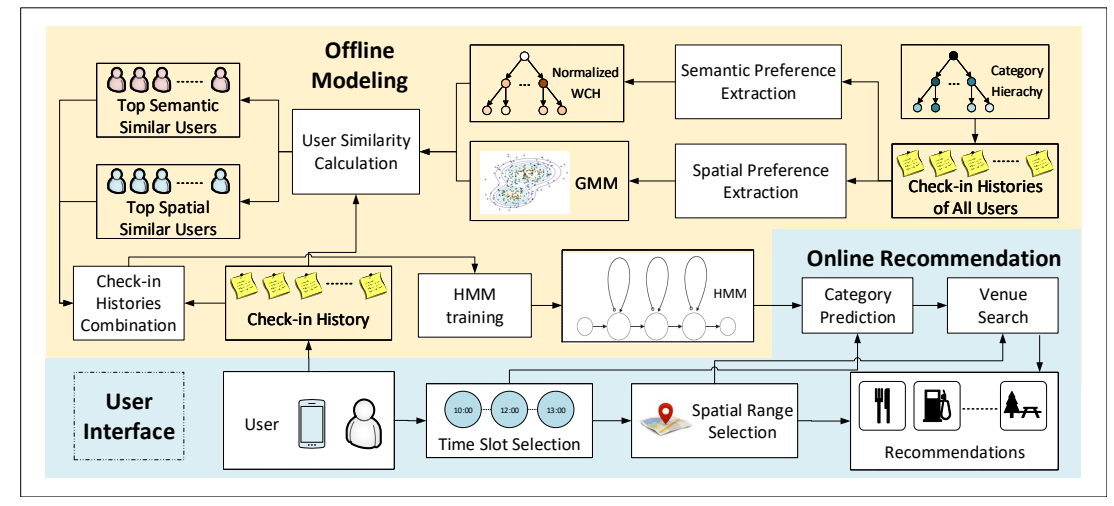

Figure 2: System Architecture.

Using GMM to model a user's check-in points is motivated by the observation that there may be several frequently visited neighborhoods for a particular user. The distribution of the check-ins from a sample user indicates some geographical clusters where she frequently checked in. Therefore, we train a GMM for each user based on her check-ins. Assume that the GMM for user $u$ is $\lambda_{u}$, and $X$ represents the feature vectors (the coordinates of check-ins) of user $v$. We can compute the average $\log$-likelihood of model $\lambda_{u}$ for the vector $X=\left\{x_{1}, \ldots, x_{T}\right\}$ using

$$
\log p\left(X \mid \lambda_{u}\right)=\frac{1}{T} \sum_{t=1}^{T} \log p\left(x_{t} \mid \lambda_{u}\right),
$$

which can be computed as the spatial similarity score from user $v$ to user $u$. By employing GMM, we are able to incorporate spatial distribution patterns into similarity score computation in order to identify the top-k most similar users given any target user.

\subsection{HMM training on categorical sequence}

A Hidden Markov Model (HMM) $[4,11]$ is a state modeling technique that deals with a situation where one observes a sequence of emissions but does not know the sequence of states the model goes through to generate those emissions. The use of HMM to model users' preference on temporal venue visit sequence is motivated by the observation that a user's next visit largely depends on her current visit.

Here we treat the categories of venues as states and the time slots as observations. As we know, a user has a higher probability to go home after she leaves her work office while a user has lower

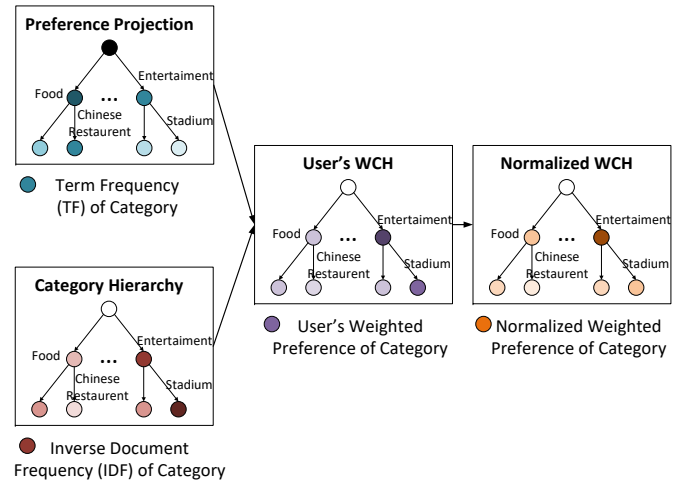

Figure 3: User WCH Construction. probability to go to a food store if she just visited a restaurant. Furthermore, the visit frequency for each venue is different. A fast food restaurant would have a higher probability to be visited at lunch time than during working hours. Therefore, each category has its own emission matrix. We train a personalized Hidden Markov Model (HMM) for time-sensitive location sequence recommendation by using the check-in sequences from both the target user and her top- $k$ most similar users in terms of both semantic and spatial similarity scores.

The notations used in HMM is shown on Table 1. The training of HMM model can be done offline and updated periodically. The training time largely depends on the number of top similar users $k$ we want to identify given any target user. In practice the $k$ value is a relatively small number because we only want to model each user's preference using a few users who are most similar to her rather than using other dissimilar users.

\section{ONLINE INFERENCE}

In this section, we discuss the online recommendation of our system, which consists of 1) category inference, which predicts the most probable category sequence for all the given time slots specified by users using Viterbi algorithm, and 2) venue search, which casts each predicted category to a specific venue (point of interest) by geophysical mapping based on users' current locations.

After training the HMM for each user, we apply Viterbi algorithm to find the most probable sequence of states (categories) that matches the time slots specified by the user. Given the model $\lambda=(A, B)$ and a sequence of observations $O=o_{1}, o_{2}, \ldots, o_{t}$, the Viterbi algorithm is able to find the most likely state sequence $S=s_{1}, s_{2}, \ldots, s_{t}$. A formal definition of the Viterbi recursion follows:

Initialization:

$$
\begin{gathered}
v_{1}(j)=a_{0 j} b_{j}\left(o_{1}\right), 1 \leq j \leq N \\
b t_{1}(j)=0
\end{gathered}
$$

Recursion:

$$
\begin{gathered}
v_{t}(j)=\underset{i=1}{\max _{i-1}} v_{t-1}(i) a_{i j} b_{j}\left(o_{t}\right), 1 \leq j \leq N, 1 \leq t \leq T \\
b t_{t}(j)=\underset{i=1}{\arg \max } v_{t-1}(i) a_{i j} b_{j}\left(o_{t}\right), 1 \leq j \leq N, 1 \leq t \leq T
\end{gathered}
$$

Termination:

$$
\text { The best score: } P *=v_{T}\left(s_{F}\right)=\max _{i=1}^{N} v_{T}(i) * a_{i F}
$$


Table 1: Notations used in Hidden Markov Model.

\begin{tabular}{|c|l|}
\hline \hline Elements & Meaning \\
\hline$S=s_{1}, s_{2}, \ldots, s_{T}$ & Hidden States, which cannot be observed directly \\
$O=o_{1}, o_{2}, . ., o_{T}$ & Observations, Can be observed directly \\
$A=a_{11}, a_{12}, \ldots, a_{n 1}, \ldots, a_{N N}$ & Transition Matrix, Describe the transfer probability among the stages \\
$B=b_{i}\left(o_{t}\right)$ & Emissions Matrix, Describe the output probability of observations in different states \\
\hline
\end{tabular}

By employing Viterbi algorithm, we are able to get the most probable category for each time slot selected by the user. For each predicted category for a particular time slot, we search for popular venues belonging to that category based on the user' current location (i.e., within a range decided by the user). When multiple categories are predicted by HMM for a particular time slot, we make multiple venue recommendations according to the probabilistic distribution of the predicted categories so that we will recommend more venues for a category with a higher visit probability.

\section{RELATED WORK}

In the last decade, learning a user's visit preferences from the user's location check-in history has attracted much attention in both academia and industry. For example, [6] stores users location histories using a matrix, where each row denotes a user and each column represents a location. In their work, the similarity between two users is simply represented by the cosine similarity between matrix rows, which do not take sequential and hierarchical properties of locations into account. In [14], hierarchical-graph-based similarity measurement is proposed to uniformly model each individual's location history and measure the similarity among users. However, it is unable to handle the data sparseness problem. [3] handles mobile users by storing a user location history into the category space and modeling user preferences using WCH. However, it does not work well for cold-start users because their calculations simply reply on counting of check-in occurrences at each venue category. For cold-start users, this operation leads to many close to zero or zero weights, making the similarity comparison perform poorly in practice. Also, [3] failed to consider user's temporal preference for venue check-ins. [5] employs GMM to capture the dynamics of human movement. [8] introduce a location recommendation framework, based on the temporal properties of user movement. Yuan et al. [13] incorporated both temporal cyclic information and geographical information for time-aware POI recommendation. There are also some studies in the literature that employ machine learning-based approaches in POI prediction or recommendation. [2] presents a system that clusters users' significant locations from GPS data and predicts location based on the Markov models. [10] uses a history of a driver's destinations to predict where a driver is going as a trip progresses. [1] designs a system based on the generation of a hidden Markov model from the past GPS log and the current location to predict a user's destination. [9] combines knearest neighbor and decision tree for predicting user's destination based on hidden Markov model. Unlike our work, all the abovementioned studies focus on the single location recommendation or prediction and therefore are unable to support the time-sensitive venue sequence recommendation. To the best of our knowledge, our work is the first attempt to address the time-aware location sequence recommendation in support of sparse user-location matrix.

\section{CONCLUSION}

In this paper, we propose a predictive framework that enables timesensitive location sequence recommendation based on sparse user check-in data on LBSNs by leveraging both mobile users' semantic and spatial similarities. The proposed framework consists of three modules: semantic similarity modeling, spatial similarity modeling, and on-line sequence recommendation. Taking full advantage of the similarities between mobile users, our proposed framework does not suffer from the cold-start problem and therefore works well even if the target user has very few footprints (check-ins).

\section{ACKNOWLEDGEMENTS}

This research has been funded in part by the National Science Foundation grants IIS-1618669 (III) and ACI-1642133 (CICI).

\section{REFERENCES}

[1] J. A. Álvarez-García, J. A. Ortega, L. G. Abril, and F. V. Morente. Trip destination prediction based on past GPS log using a hidden markov model. Expert Syst. Appl., 37(12):8166-8171, 2010.

[2] D. Ashbrook and T. Starner. Using GPS to learn significant locations and predict movement across multiple users. Personal and Ubiquitous Computing, 7(5):275286, 2003.

[3] J. Bao, Y. Zheng, and M. F. Mokbel. Location-based and preference-aware recommendation using sparse geo-social networking data. In SIGSPATIAL 2012 International Conference on Advances in Geographic Information Systems (formerly known as GIS), SIGSPATIAL'12, Redondo Beach, CA, USA, November 7-9, 2012, pages 199-208, 2012.

[4] L. E. Baum and T. Petrie. Statistical inference for probabilistic functions of finite state markov chains. Ann. Math. Statist., 37(6):1554-1563, 121966.

[5] E. Cho, S. A. Myers, and J. Leskovec. Friendship and mobility: user movement in location-based social networks. In Proceedings of the 17th ACM SIGKDD International Conference on Knowledge Discovery and Data Mining, San Diego, CA, USA, August 21-24, 2011, pages 1082-1090, 2011.

[6] C. Chow, J. Bao, and M. F. Mokbel. Towards location-based social networking services. In Proceedings of the 2010 International Workshop on Location Based Social Networks, LBSN 2010, November 2, 2010, San fose, CA, USA, Proceedings, pages 31-38, 2010.

[7] B. Fuglede and F. Topsoe. Jensen-shannon divergence and hilbert space embedding. In International Symposium onInformation Theory, 2004. ISIT 2004. Proceedings., pages 31-, June 2004.

[8] H. Gao, J. Tang, X. Hu, and H. Liu. Exploring temporal effects for location recommendation on location-based social networks. In Seventh ACM Conference on Recommender Systems, RecSys '13, Hong Kong, China, October 12-16, 2013, pages 93-100, 2013.

[9] Y. Kim and S. Cho. A hmm-based location prediction framework with location recognizer combining k-nearest neighbor and multiple decision trees. In Hybrid Artificial Intelligent Systems - 8th International Conference, HAIS 2013, Salamanca, Spain, September 11-13, 2013. Proceedings, pages 618-628, 2013.

[10] J. Krumm and E. Horvitz. Predestination: Inferring destinations from partial trajectories. In UbiComp 2006: Ubiquitous Computing, 8th International Conference, UbiComp 2006, Orange County, CA, USA, September 17-21, 2006, pages 243-260, 2006.

[11] L. R. Rabiner and B. H. Juang. An introduction to hidden Markov models. IEEE ASSP Magazine, pages 4-15, January 1986.

[12] W. Wang and W.-S. Ku. Recommendation-based smart indoor navigation. In Internet-of-Things Design and Implementation (IoTDI), 2017 IEEE/ACM Second International Conference on, pages 311-312. IEEE, 2017.

[13] Q. Yuan, G. Cong, Z. Ma, A. Sun, and N. Magnenat-Thalmann. Time-aware pointof-interest recommendation. In The 36th International ACM SIGIR conference on research and development in Information Retrieval, SIGIR '13, Dublin, Ireland fuly 28 - August 01, 2013, pages 363-372, 2013.

[14] Y. Zheng, L. Zhang, Z. Ma, X. Xie, and W. Ma. Recommending friends and locations based on individual location history. TWEB, 5(1):5:1-5:44, 2011. 